Original paper

\title{
Beamline characterization of a dielectric-filled reentrant cavity resonator as beam current monitor for a medical cyclotron facility
}

\author{
Sudharsan Srinivasan*, Pierre-André Duperrex, Jacobus Maarten Schippers \\ Paul Scherrer Institute (PSI), Villigen, Switzerland
}

\section{A R T I C L E I N F O}

\section{Keywords:}

Proton therapy

Beam current monitor

Cavity resonator

Energy spread

Sensitivity

\begin{abstract}
A B S T R A C T
At PSI (Paul Scherrer Institute), Switzerland, a superconducting cyclotron called "COMET" delivers proton beam of $250 \mathrm{MeV}$ pulsed at $72.85 \mathrm{MHz}$ for proton radiation therapy. Measuring proton beam currents (0.1-10nA) is of crucial importance for the treatment safety and is usually performed with invasive monitors such as ionisation chambers (ICs) which degrade the beam quality. A new non-invasive beam current monitor working on the principle of electromagnetic resonance is built to replace ICs in order to preserve the beam quality delivered. The fundamental resonance frequency of the resonator is tuned to $145.7 \mathrm{MHz}$, which is the second harmonic of the pulse rate, so it provides signals proportional to beam current. The cavity resonator installed in the beamline of the COMET is designed to measure beam currents for the energy range 238-70 MeV. Good agreement is reached between expected and measured resonator response over the energy range of interest. The resonator can deliver beam current information down to $0.15 \mathrm{nA}$ for a measurement integration time of $1 \mathrm{~s}$. The cavity resonator might be applied serving as a safety monitor to trigger interlocks within the existing domain of proton radiation therapy. Low beam currents limit the abilities to detect sufficiently, however, with the potential implementation of FLASH proton therapy, the application of cavity resonator as an online beam-monitoring device is feasible.
\end{abstract}

\section{Introduction}

The PROSCAN facility at PSI, dedicated for proton therapy consists of COMET [1,2], which is a $250 \mathrm{MeV}$ superconducting cyclotron followed by a degrader (238-70 MeV) and with beamlines leading to three gantries and a specific area for eye irradiations. The PROSCAN incorporates checkpoints to comply with safe operation of the beam based on certain beam parameters such as current, position, energy etc. This set of information is obtained using dedicated beam monitoring systems. The beam current plays hereby a critical role as it is directly linked to the dose-rate applied to the patient, therefore, requiring accurate and precise determination during standard operation in a particle therapy facility [3]. This puts a demand on the diagnostics to deliver highly accurate signals with minimum beam disturbance [4]. The beam current beyond the degrader is in the range of $0.1-10 \mathrm{nA}$ (where $1 \mathrm{nA}$ at $72.85 \mathrm{MHz}$ corresponds to approximately $0.01 \mathrm{fC}$ / bunch). The beam current is predominantly measured with the help of planar ICs [5,6] which is a beam intercepting method [7], leading to scattering, energy loss and activation of the detector itself $[8,9]$.

To prevent these effects from an IC, the application of a positionindependent non-invasive beam current monitor is conceived. Most of the non-invasive beam current monitors employ either the electric or the magnetic field of the charged particle beam [10] for detection of the beam current on a broadband basis. Some of the already existing noninvasive beam current monitors have been used for detection of lowlevel bunch charge (down to few hundred $f C$ ), however not down to $0.01 \mathrm{fC}$ or an average beam current of $1 \mathrm{nA}$ especially in the energy range of $238-70 \mathrm{MeV}$.

For instance, monitors that couple to the electric field such as a capacitive pickup of the button type in Argonne Wakefield Accelerator (AWA) facility are limited in signal detection down to $100 \mathrm{fC}$ [11]. Moreover, their signal resolution is limited by the horizontal-vertical coupling between the individual pickups [12]. Stripline monitors provide better signal level than conventional button type monitors at the expense of a complex mechanical realization such as in the DESY Tesla Test Facility, Phase 2 (TTF2) for the electron linac [12].

The beam diagnostics that couple to the magnetic field of the beam such as Fast Current Transformers (FCTs) are limited in measurement due to their detection threshold of several tens of $\mu \mathrm{A}$ [13]. This detection threshold is a result of the large bandwidth of these monitors in the range of several $\mathrm{kHz}$ to almost $1 \mathrm{GHz}$. Moreover, at higher frequencies these FCTs become sensitive to both beam positon with $>1 \%$ / $\mathrm{mm}$ and a bunch length dependence of $1 \%$ thus affecting the signal sensitivity as shown in [14]. Integrated Current Transformer (ICT) from

\footnotetext{
* Corresponding author at: Forschungstrasse 111, WBGA/C33, 5232 Villigen PSI, Switzerland.

E-mail address: sudharsan.srinivasan@psi.ch (S. Srinivasan).
} 
Table 1

Extracted beam from the COMET cyclotron and beyond the degrader.

\begin{tabular}{ll}
\hline Beam properties & Units \\
\hline Extracted beam current & $<1-1000 \mathrm{nA}$ \\
Energy spread $\Delta \mathrm{E} / \mathrm{E}$ at extraction & $0.15 \%$ \\
$\Delta \mathrm{E} / \mathrm{E}$ Beyond the degrader & $0.2 \%$ at $230 \mathrm{MeV} ; 2.5 \%$ at $70 \mathrm{MeV}$ \\
Beam current beyond degrader & $0.1-10 \mathrm{nA}$ \\
Beam diameter & $1-20 \mathrm{~mm}$ \\
Repetition rate & $72.85 \mathrm{MHz}(\mathrm{T}=13.73 \mathrm{~ns})$ \\
Bunch length and charge at degrader exit for & $2 \mathrm{~ns} ; \approx 0.01 \mathrm{fC}$ (for $1 \mathrm{nA})$ \\
$\quad$ & \\
\hline
\end{tabular}

Bergoz instrumentation [15]; provides better signal sensitivity compared to conventional FCTs. However, these are limited in detection to sub-micro ampere beam current for continuous waveform (cw) beams.

Inductive pickups, which are used for broadband bunch observations, to measure beam image current are limited by parasitic inductances at low frequencies for instance at the Drive Beam Linac of the Third CLIC Test Facility (CTF3 DBL) at CERN [16]. Wall Current Monitors (WCM) [17], due to their broadband capability, will have a higher thermal noise level which will limit their sensitivity to measure low beam currents of 0.1-10nA in the PROSCAN beamlines.

In order to improve the detection threshold and the sensitivity of the beam current measurement with broadband monitors, one can implement analog narrowband processing of the signals at a single harmonic of the acceleration frequency. This aids in a significant reduction of the thermal noise [12]. However, the output signal becomes bunch shape dependent, leading to relative beam current measurements. This is a concern for cavity measurements as well.

Cavity resonators, unlike broadband monitors, measure signal from a single harmonic of the bunch spectrum. These cavities in their fundamental mode, i.e. Transverse Magnetic mode [18] are used more commonly to measure small beam currents due to their high sensitivity to measure in the range of few nA [10]. These are typically used as precise position monitors in Free Electron Laser (FEL) [19] facilities where the pulse length is typically in the order of $1 \mu$ s.

Even though cavity resonators [20-22], provide less signal information than from a typical FCT, it has been chosen as the beam current monitor of choice as they are expected to provide significant noise reduction and mode separation. Moreover, the quality factor of the cavity can be made sufficiently larger compared to a typical oscillating circuit and the risk related to electromagnetic interference are significantly lower for a cavity that is traversed by the beam. These are important design criteria due to the low signal strength in beamlines.

Here we describe a cavity resonator that has been developed [23] at PSI. It works on the fundamental mode tuned to $145.7 \mathrm{MHz}$, which is the second harmonic of the bunch repetition rate. In previous measurements $[23,24]$, we have studied the feasibility of the cavity resonator and have validated the prototype on a stand-alone test-bench. We will also report on the investigation of the beamline performance of the cavity resonator as a low-current beam current monitor. The design considerations for the cavity resonator are briefed, followed by a description of the effects of the proton beam energy spread, the measurement chain, and the signal offset on the measured signal. The measured parameters, i.e. the sensitivity and the measurement offset are compared with expectation. This work discusses the potential advantages and disadvantages of the cavity resonator as a non-invasive monitor for low-energy low-current proton beams. The properties of the beam that are of interest to this paper are given in Table 1.

\section{Materials and methods}

\section{1. $T M_{O 10}$ cavity resonator}

A dielectric-filled reentrant cavity resonator, modelled as lumped element LC resonator, is built to measure beam currents for the beamlines as shown in Fig. 1. This helps to keep the induced electric and magnetic fields concentrated separately in region 2 and 3 as shown in Fig. 1. These induced fields are governed by the outer metallic boundary such that they sustain a discrete set of resonance frequencies with their characteristic field distribution [22]. The fundamental mode i.e. $\mathrm{TM}_{\mathrm{mnp}}=\mathrm{TM}_{010}$ mode has its field components in the azimuthal direction $(\varphi)$ with zero $(\mathrm{m}=0)$ full-period variations, in the radial direction (r) with one zero of the axial field component $(n=1)$ and in the longitudinal direction $(\mathrm{z})$ with zero half-period variations $(\mathrm{p}=0)$. Within a pillbox cavity, a pure $\mathrm{TM}_{010}$ mode can be excited.

The system developed at PSI is smaller (See Fig. 2) compared to a pillbox equivalent, which helps in precise and simpler manufacturing. Moreover, with appropriate sizing of the gap, stronger damping of the higher order modes can be achieved. This minimizes the contribution of these modes to measurements performed at the $\mathrm{TM}_{010}$ resonance frequency [21].

Insertion of a dielectric ring made of Macor [25] in the reentrant gap lowers the unloaded quality factor $Q_{o}$ of the cavity resonator. This lowers the measurable quality factor $\mathrm{Q}_{\mathrm{L}}$ whose inverse value is defined by the sum of the individual inverses of the external quality factor $Q_{e x}$
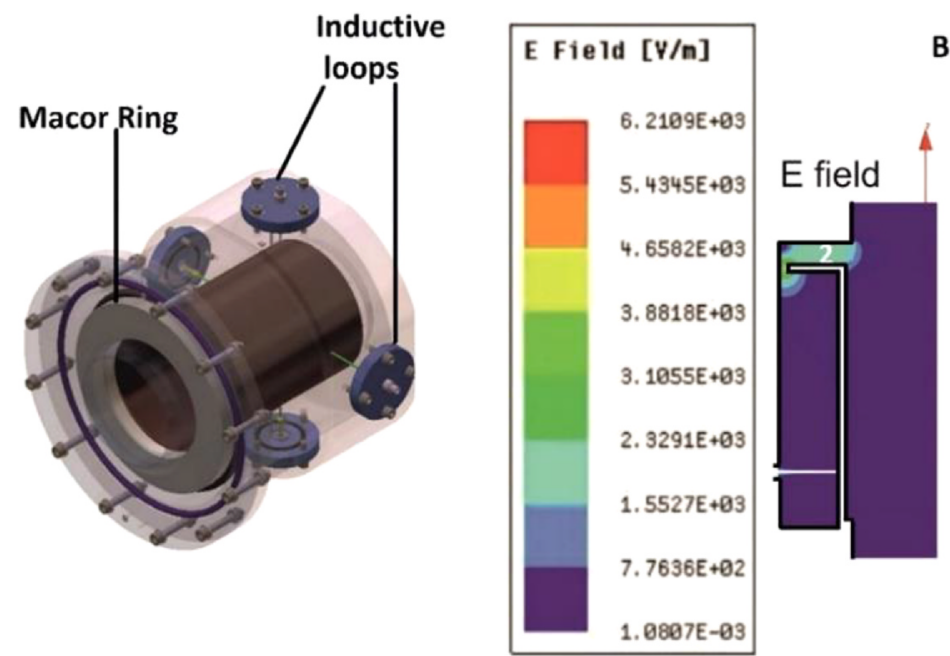

Beam Axis

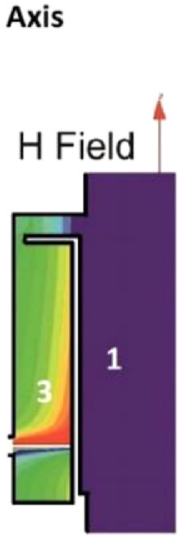

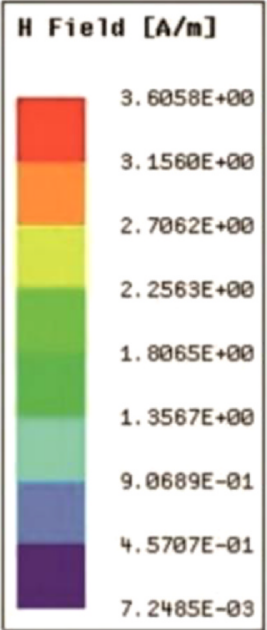

Fig. 1. 3-dimensional view of the reentrant cavity resonator (left) with markings of the inductive loops and MACOR ring. The E fields and $\mathrm{H}$ fields excited within the resonator shows the separation of the capacitive (marked as 2) and inductive zones (marked as 3 ) in this resonator (right). The field plots show the absolute values of the induced fields for a cavity stored energy of $1 \mathrm{~J}$. 


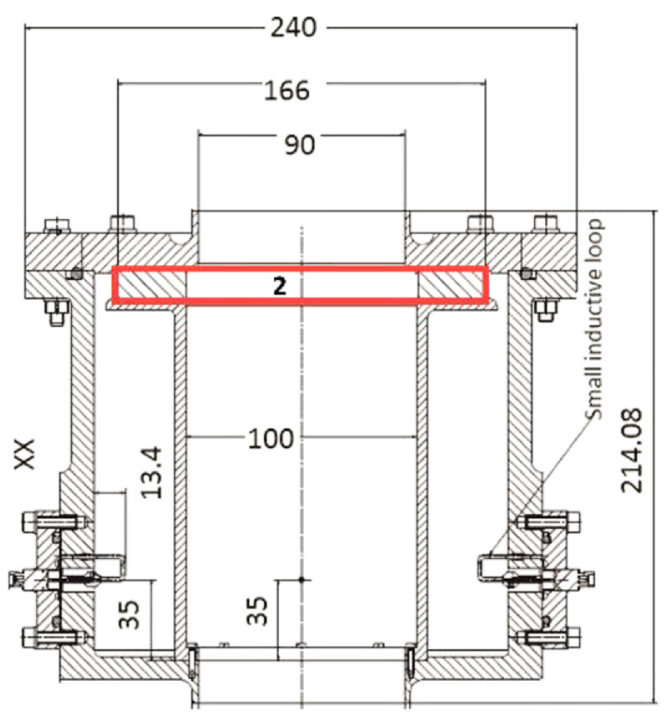

All dimensions are in $\mathrm{mm}$
Beam tube/inner coaxial(region 1)

Dielectric filling/capacitive zone (region 2)

Outer coaxial/inductive zone (region 3)

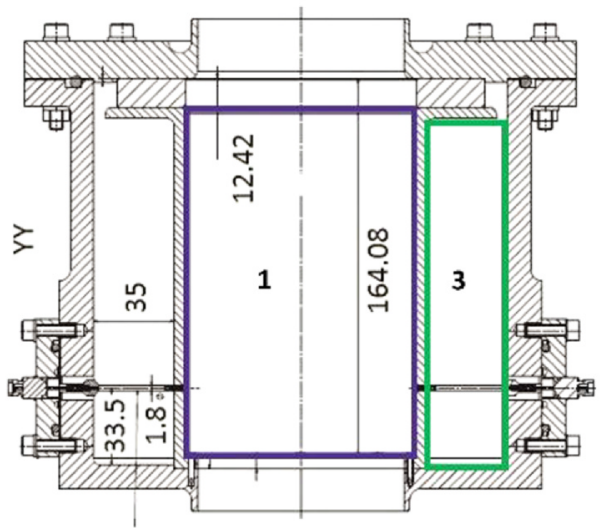

Large inductive loop

Fig. 2. Cut-section of the reentrant cavity resonator ( $\mathrm{XX}$ and $\mathrm{YY}$ ) with dimensions correpsonding to $\mathrm{TM}_{010}$ frequency at $145.7 \mathrm{MHz}$. Region 1,2 and 3 represent the inner coaxial (or beam tube), dielectric filling in the reentrant gap (capacitive zone), and the outer coaxial (inductive zone).

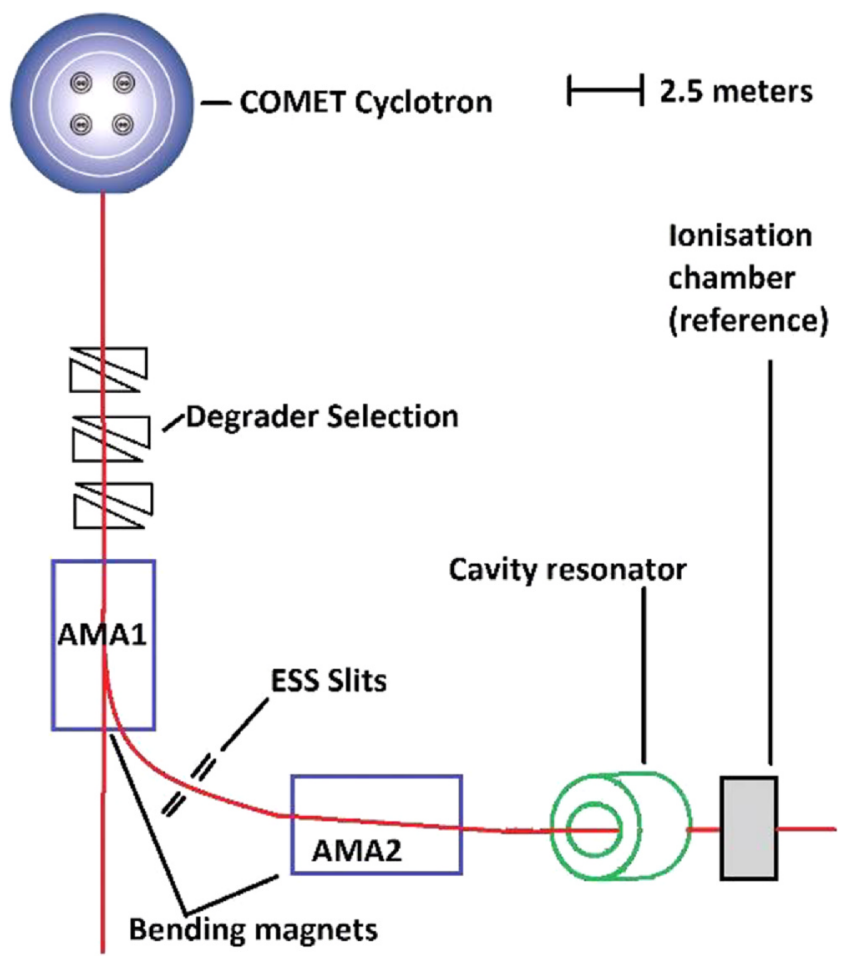

Fig. 3. Location of the cavity resonator in the PROSCAN layout. Marked are the COMET cyclotron, degrader selection, ESS for momentum spread, bending magnets, cavity resonator and the IC which is used as beam current reference.

and the unloaded quality factor $\mathrm{Q}_{\mathrm{o}}$ as given in [23]. A low measurable quality factor helps in minimizing the detune of the $\mathrm{TM}_{010}$ resonance frequency for small temperature changes [20].

The cavity resonator is provided with four inductive loops (two small and two large loops), where one large loop is dedicated for beam current measurements and the other as a resonance trombone for tuning purposes. The pair of small loops is used for verification of the design frequency when connection issues exist [23]. The presence of multiple measurement ports lowers the external quality factor [26] which results in higher output signal level as discussed in [21]
Table 2

Estimate of the second harmonic amplitude factor for a rectangular bunch shape [33] at the resonator location for different beam energies. Bunch length of $2 \mathrm{~ns}$ at the degrader exit is assumed as reference for the calculation.

\begin{tabular}{lllll}
\hline Energy, MeV & $\begin{array}{l}\text { Energy } \\
\text { spread, } \\
\text { MeV }\end{array}$ & $\begin{array}{l}\text { Momentum } \\
\text { spread, \% }\end{array}$ & $\begin{array}{l}\text { Bunch length } \\
\text { at resonator, ns }\end{array}$ & $\begin{array}{l}\text { Second harmonic } \\
\text { amplitude factor, } \\
\mathrm{A}_{2}\end{array}$ \\
\hline 79 & 4.4 & 1.0 & 3.24 & 0.67 \\
109 & 4.2 & 1.0 & 3.08 & 0.69 \\
139 & 3.9 & 1.0 & 2.97 & 0.71 \\
171 & 3.3 & 1.0 & 2.91 & 0.73 \\
201 & 2.7 & 0.84 & 2.71 & 0.76 \\
231 & 1.7 & 0.70 & 2.56 & 0.79 \\
\hline
\end{tabular}

$\mathrm{V}_{\text {out }}=\sqrt{2 \mathrm{ZP} \mathrm{P}_{\mathrm{ex}}} \equiv \omega_{010} \mathrm{q} \sqrt{\frac{\mathrm{Z}}{\mathrm{Q}_{\mathrm{ex}}}\left(\frac{\mathrm{R}}{\mathrm{Q}_{\mathrm{o}}}\right)_{010}} \mathrm{e}^{\left(\frac{-\omega_{010}^{2} \sigma_{\mathrm{Z}}^{2}}{2 \beta^{2} \mathrm{c}^{2}}\right)}$

where $\mathrm{V}_{\text {out }}$ is the output voltage, for a given bunch length $\sigma_{z}$ (in meters) measured over an impedance, $\mathrm{Z}=50 \Omega$. $\mathrm{P}_{\mathrm{ex}}$ is the output power from the cavity, for $\mathrm{q}$ as the bunch charge, at the resonance frequency $\omega_{010}$, whose normalised shunt impedance is $\left(\mathrm{R} / \mathrm{Q}_{\mathrm{o}}\right)_{010}$ which is a figure of merit for the shape of the cavity [20].

The expected voltage measured with a large inductive loop over $50 \Omega$ impedance for a beam in $2 \mathrm{~ns}$ bunch and an average current of $1 \mathrm{nA}$ is approximately $15 \mathrm{nV}$ [23]. For the resonator to deliver the same sensitivity independent of the beam energy, the resonator must be located not further downstream of the degrader. Otherwise, with decreasing energy, bunch length is increased due to the degrader's energy spread contributions. This is expected to lower the amplitude of the second harmonic component in the bunch, which will reduce the resonator sensitivity for lower energies. Thus, to measure the beam current in the energy range 238-70 $\mathrm{MeV}$ with an energy independent sensitivity, location of the resonator should be within close proximity from the degrader exit.

\subsection{Cavity location in PROSCAN facility and effect of bunch length}

The PROSCAN facility is temperature controlled $\left(28.5 \pm 0.5{ }^{\circ} \mathrm{C}\right)$, in order to have stable operating conditions for its beamline elements. In PROSCAN, delivery of multiple beam energies in the range $238-70 \mathrm{MeV}$ 


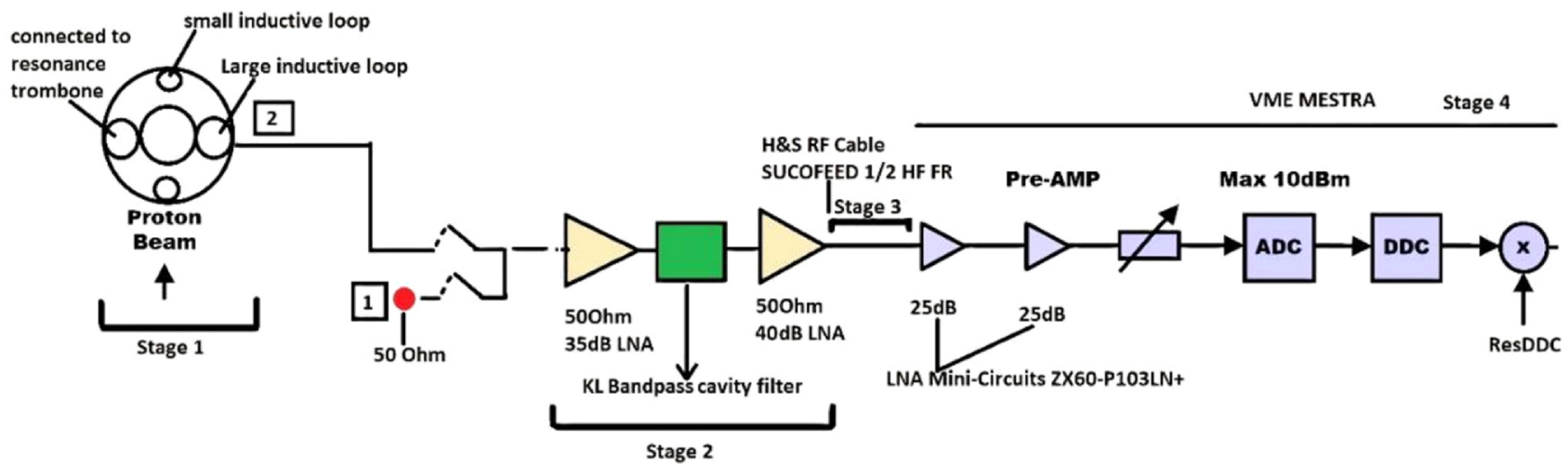

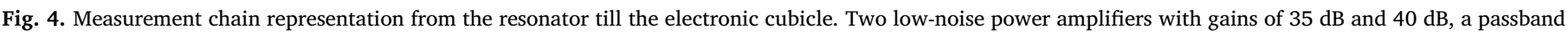

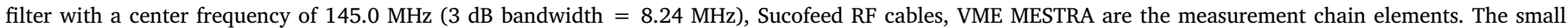

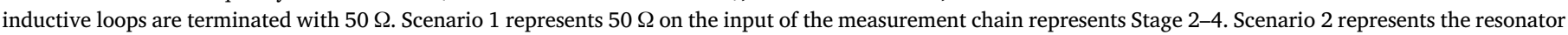
connected to the measurement chain represents Stage 1-4.

Table 3

Power budget of the measurement system for 1nA beam current as excitation for the cavity resonator.

\begin{tabular}{llll}
\hline Stage & measurement system elements & Gain $(\mathrm{dB})$ & $\begin{array}{l}\text { Cumulative power level } \\
(\mathrm{dBm})\end{array}$ \\
\hline 1 & Resonator & - & -143.0 \\
2 & Local amplifiers & +75.0 & -68.0 \\
3 & $1 / 2 "$ H\&S SUCOFEED cable & -3.0 & -71.0 \\
& $50 m$ & & -21.0 \\
4 & VME MESTRA total & +50.0 & \\
& amplification & & \\
\hline
\end{tabular}

is achieved by means of a carbon wedge degrader [27]. The degradation process of the beam results in growth of emittance and energy spread [28-30]. To have the required beam quality at the patient location, the beam is shaped by a set of collimators and an Energy Selection System (ESS) that helps in reducing the energy spread. An emittance of $30 \pi \mathrm{mm}$ mrad and $\pm 1.0 \%$ of momentum spread is allowed to match the acceptance of beamlines and gantries [27]. The effect of energy spread increase leads to an energy dependent decrease in bunch amplitude and an increase in bunch length down the beamline in the beam current. Thus at $16 \mathrm{~m}$ from the degrader where the cavity resonator is positioned as marked in Fig. 3, the second harmonic of the beam repetition rate is reduced because of these effects.

For beamline validation of the cavity resonator, multiple energies are chosen as given in Table 2. The energy spread induced by the degrader at these energies is calculated as per [31] and is within acceptable range of the measured energy spread as given in [32]. For energies lower than $180 \mathrm{MeV}$, the ESS helps in reducing the momentum spread to $\pm 1 \%$, but at the energies $231 \mathrm{MeV}$ and $201 \mathrm{MeV}$, the momentum spread is smaller than $1 \%$ and therefore not limited by the ESS.

The bunch length at the location of the resonator is estimated from the momentum spread for different energies. Due to the momentum selection in the ESS, a rectangular distribution can be assumed for the shape of the beam bunches. The amplitude of the lower spectral components of the bunch time structure is approximated by a Sinc function that corresponds to a Fourier transform of a square pulse. The estimate of the second harmonic amplitude factor, $\mathrm{A}_{2}$, which is normalised to the average beam current, is calculated [33] which decreases with

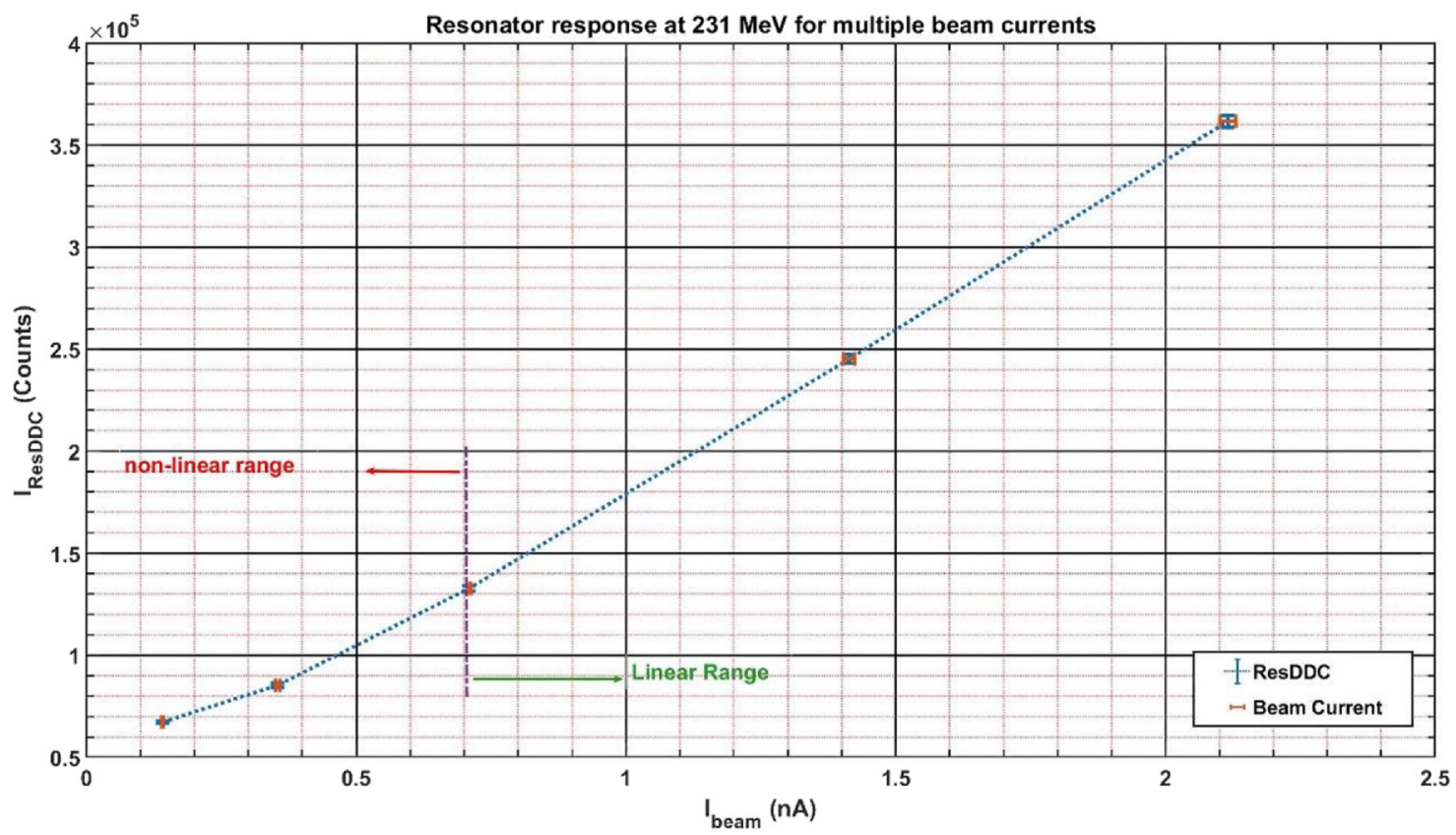

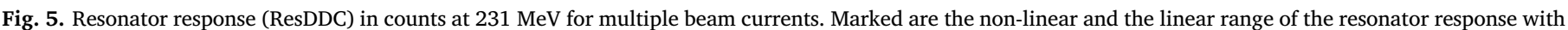
beam current. The dotted line represents the trend of the measurement. 


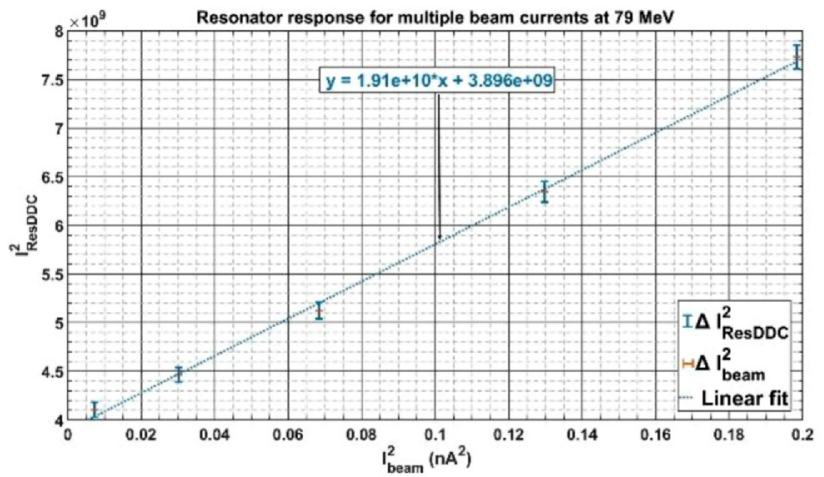

(a)

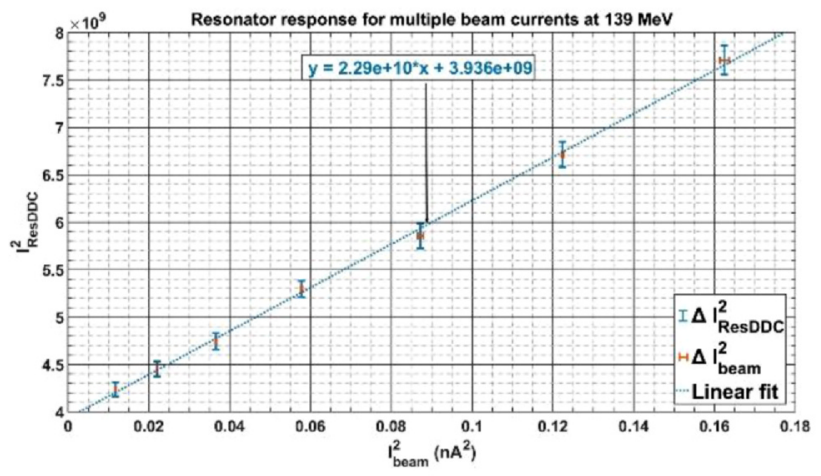

(c)

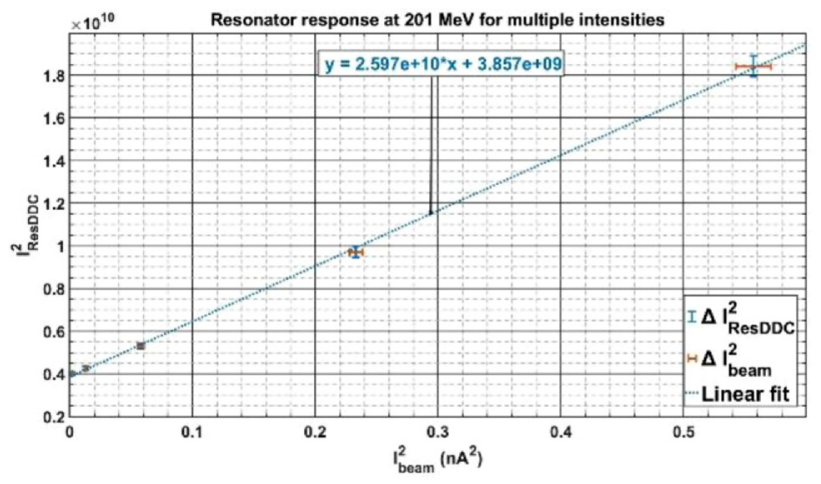

(e)

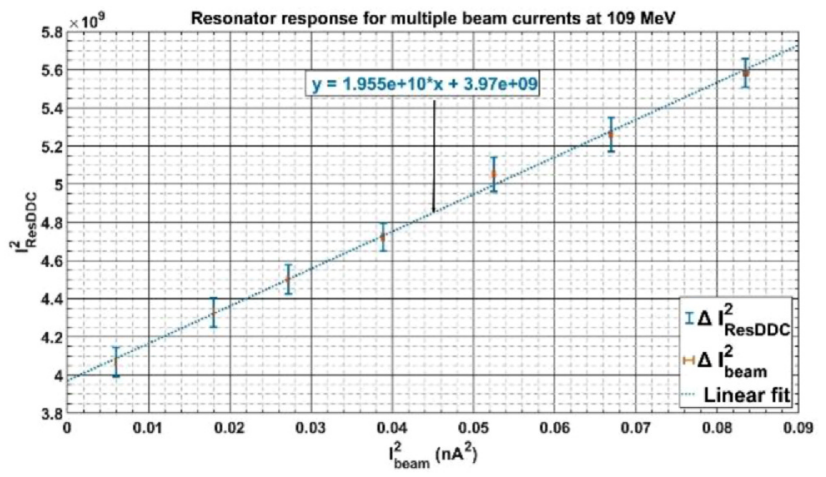

(b)

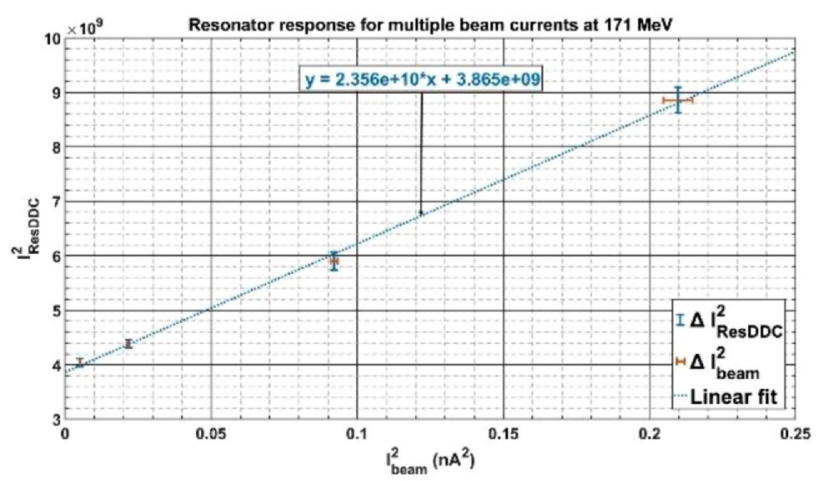

(d)

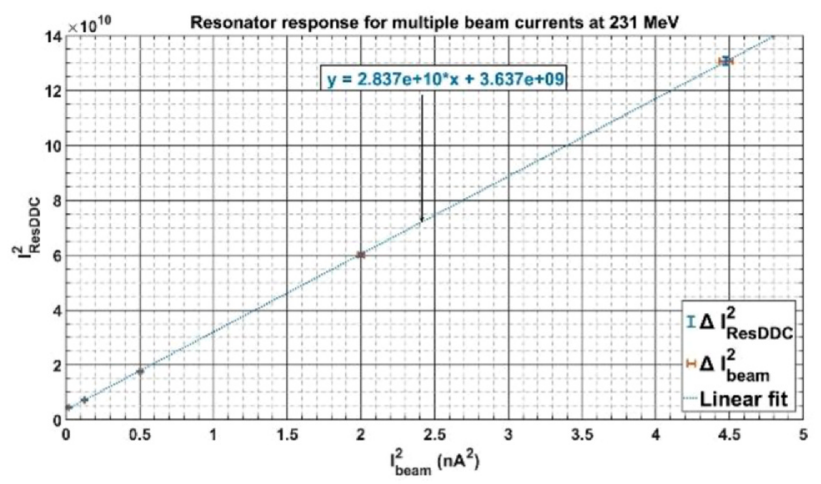

(f)

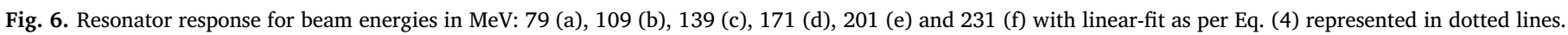

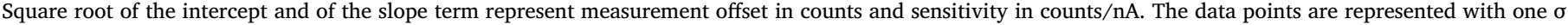
deviation. The dotted line represents the linear fit.

increasing bunch length as given in Table 2.

\subsection{Measurement chain}

For the beam current measurements with the cavity resonator, a PSI developed measurement system called VME MESTRA is used [34]. The measurement system is configured to convert the amplitude of the 145.7 MHz RF-signal from the cavity into a proportional voltage signal. The measurement chain as shown in Fig. 4 starts with a measurement port i.e. a large inductive loop of the cavity resonator. The other large inductive loop is connected to a resonance trombone for tuning capabilities. The pair of the small inductive loops are terminated with $50 \Omega$. The cavity signal is amplified with a low-noise amplifier of $35 \mathrm{~dB}$ gain [35]. The amplified signal is then bandpass filtered with a customized cavity type filter from KL microwave. The bandpass filter has its center frequency at $145.0 \mathrm{MHz}$ with a $3 \mathrm{~dB}$ bandwidth of $8.24 \mathrm{MHz}$. The filtered signal is further amplified by a $40 \mathrm{~dB}$ gain with a FEMTO wideband low-noise amplifier [36]. The filtered and amplified signals from the cavity enter the main sub-system of the measurement system whose components are:

- $2 \mathrm{X} 25 \mathrm{~dB}$ preamplifier [37]

- 16-bit Digitizer (ADC3110) [38] with 50.0 MSamples/s sampling rate, maximum input power $10 \mathrm{dBm}$

- Digital Down Converter (DDC)

The digitized signal is down-converted through a Field Programmable Gate Array (FPGA) on the Digital Down Converter (DDC). The DDC results in an output signal, Res DDC, with $50 \mathrm{k}$ samples/s following a decimation with a factor of 1000 from the input 
Table 4

Measurement summary for resonator at different energies. The fractional uncertainty of the evaluated measurement offset and the resonator calibration factor are derived from the in-beam measurements through error propagation. The value of the normalizing term, $\mathrm{C}=212042$ counts $/ \mathrm{nA} . \mathrm{A}_{2}$, is taken from Table 2 .

\begin{tabular}{llll}
\hline $\begin{array}{l}\text { Proton } \\
\text { beam } \\
\text { energy } \\
(\mathrm{MeV})\end{array}$ & $\begin{array}{l}\text { Measured resonator } \\
\text { sensitivity, k_meas } \\
( \pm 1.35 \%) \text { (counts) }\end{array}$ & $\begin{array}{l}\text { Expected resonator } \\
\text { sensitivity, } \mathrm{k} \\
\left(=\mathrm{A}_{2} * \mathrm{C}\right)(\text { counts) } \\
\mathrm{nA})\end{array}$ & $\begin{array}{l}\text { Measurement offset, } \\
\mathrm{I}_{\text {measoff }}( \pm 1.26 \%) \\
\text { (counts) }\end{array}$ \\
\hline 79 & 138,202 & 142,068 & 62,418 \\
109 & 139,821 & 146,309 & 63,008 \\
139 & 151,327 & 150,550 & 62,737 \\
171 & 153,493 & 154,791 & 62,169 \\
201 & 161,152 & 161,152 & 62,104 \\
231 & 168,434 & 167,513 & 60,308 \\
\hline
\end{tabular}

signal. The DDC filters the input noise power of the digitized signal with its $18 \mathrm{kHz}(3 \mathrm{~dB})$ bandwidth in order to get a high attenuation for the stop band and a flat pass band. For a beam current of $1 \mathrm{nA}$ at the resonator location, the power budget of the measurement system is given in Table 3.

\section{Results}

\subsection{No beam response with and without resonator}

Prior to in-beam characterization of the cavity resonator, the no beam response is measured without resonator (scenario 1 marked in Fig. 4) and with the resonator (scenario 2 marked in Fig. 4) in the measurement chain. This measurement was performed when the cyclotron RF was switched on but with no beam traversing the through the monitor for both the scenarios.

The measurement with no resonator and a $50 \Omega$ termination on the measurement cable (scenario 1, Fig. 4) was recorded as 40,000 counts with the standard deviation of $1.26 \%$. The measurement offset, which is the no beam resonator response (scenario 2, Fig. 4) is recorded as $I_{\text {measoff }}=63007$ counts with a standard deviation of $1.26 \%$ i.e. 800 counts. This is representative of the noise floor of the measurement chain including the resonator and its RF interferences. The difference between the two values is the amplitude of the RF interference in counts.

\subsection{In-beam resonator response}

The resonator response is measured for beam current sweeps in the range 0-2.5 nA for the beam energies given in Table 2. For the calibration of the cavity resonator as a beam current monitor, an IC $[5,6]$ is used as a reference monitor, as marked in Fig. 3. The reference monitor was chosen immediately behind the resonator in order to have same beam current amplitude for comparison. The measured resonator response with respect to the beam current at $231 \mathrm{MeV}$ is shown in Fig. 5. The standard deviation of the measured response at every beam current is approximately 800 counts.

Ideally, for high beam current range as marked in Fig. 5, the relation between the resonator response given as $\mathrm{I}_{\text {ResDDC }}$ in counts and the absolute beam current given as $\mathrm{I}_{\text {beam }}$ in $\mathrm{nA}$ is considered linear

$\mathrm{I}_{\text {ResDDC }}=\mathrm{kI}_{\text {beam }}$

where $\mathrm{k}$ is the resonator sensitivity whose unit is counts/nA. On the contrary, for lower beam current range as marked in Fig. 5, the relation between $I_{\text {ResDDC }}$ and the $I_{\text {beam }}$ is non-linear since the $I_{\text {ResDDC }}$ is within the range of the measurement offset, $I_{\text {measoff. A linear relationship is }}$ established for the lower beam current range given by

$\mathrm{I}_{\text {ResDDC }}^{2}=\mathrm{I}_{\text {measoff }}^{2}+\mathrm{k}^{2} \mathrm{I}_{\text {beam }}^{2}$

where the power of the measured signal $\left(\mathrm{I}_{\text {ResDDC }}^{2}\right)$ is the sum of the individual power of the measurement offset $\left(\mathrm{I}_{\text {measoff }}^{2}\right)$ and power of the beam current response $\left(\mathrm{k}^{2} \mathrm{I}_{\text {beam }}^{2}\right)$. Such a relation is possible since the beam current response and the measurement offset are uncorrelated.

Fig. 6 represents the beam current measurement represented in power form i.e. $I_{\text {ResDDC }}^{2}$ with respect to the power of the beam current i.e. $\mathrm{I}_{\text {beam }}^{2}$ for all energies. The linear fit is of the form as represented in Eq. (3) where the intercept term of the linear fit equations is the power of the measurement offset $\mathrm{I}_{\text {measoff }}^{2}$ and the slope term is square of the resonator sensitivity i.e. $\mathrm{k}^{2}$. The measurement offset and the resonator

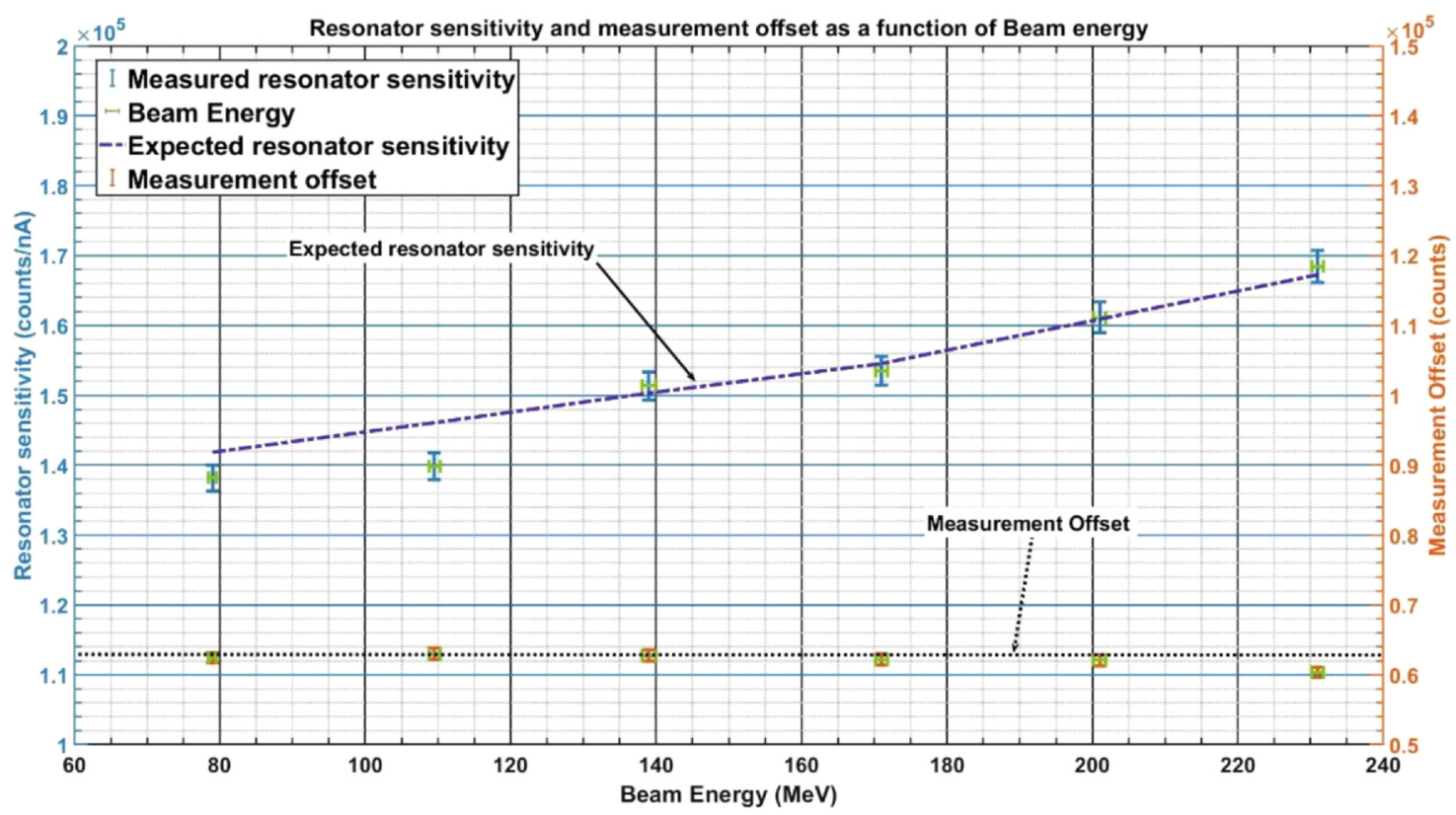

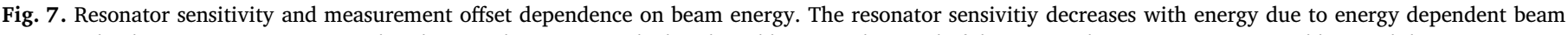

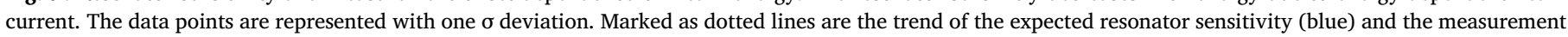

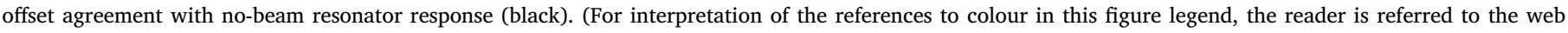
version of this article.) 
sensitivity are given as square root of the intercept term and of the slope term from Fig. 6 and are summarized in Table 4.

The measurement offset derived from the fit intercept term for all energies is within acceptable range of the no beam resonator response i.e. 63,007 counts. This indicates that there has been no presence of unexpected noise contributions during the measurement.

The measured resonator sensitivity, k_meas (in units of counts/nA), given in Table 4, decreases with beam energy as expected (i.e. expected resonator sensitivity, $\mathrm{k}$ ) due to an energy dependent decrease in the beam current. This trend is clearly observable in Fig. 7. The expected sensitivity, $\mathrm{k}$, is within one standard deviation $(\sigma)$ of the measured sensitivity, k_meas, for all energies except for $109 \mathrm{MeV}(\approx 3 \sigma)$ and $79 \mathrm{MeV}(\approx 2 \sigma)$ which could be due to higher corrections applied for the beam losses. The product of the second harmonic amplitude factor $\mathrm{A}_{2}$, (from Table 2) with a normalizing term, C, provides the expected resonator sensitivity, $\mathrm{k}$, in Table 4 . The value of the normalizing term, $\mathrm{C}=212042$ counts/nA ( $\mathrm{C}=161152 / 0.76)$ is derived by comparing the measured sensitivity, $\mathrm{k}_{-}$meas with $\mathrm{A}_{2}$ of $201 \mathrm{MeV}$. The reason is that $201 \mathrm{MeV}$ is one of the energies where the ESS does not alter the momentum spread induced by the degrader, thus serving as a more reliable reference for correlating.

The measurement offset and the sensitivity derived from the inbeam measurements are considered reasonable as they are in good agreement with the no beam measurement offset and the expected sensitivity. Thus, the linear-fit equations given in Fig. 6 are used to determine the lowest detectable beam current. Moreover, the measurements from the cavity is considered position independent as the position dependence is approximately $0.03 \% / \mathrm{mm}$ measured at $60 \%$ of the beam pipe radius. This is due to the fact the Bessel function of the order $m=0$ is independent of the radius close to the cavity center [39]. Thus, cavity resonators serve better for beam current measurement compared to FCTs whose position dependence is $>1 \% / \mathrm{mm}$ [14].

We defined the lowest detectable beam current by the condition when the resonator response (i.e. ResDDC) is minimum three $\sigma$ (defined resolution) higher than their measurement offset. This condition provides the lowest detectable beam current as $0.15 \mathrm{nA}$ with a resolution of $0.05 \mathrm{nA}$ for all energies as derived from the fit equations given in Fig. 6 .

\section{Discussions}

In this work, we have measured the resonator sensitivity, k_meas and the measurement offset, $I_{\text {measoff }}$ for energies in the range 238-70 MeV. The highlight of the measurement is the good agreement of the above measurement quantities, $\mathrm{k}_{-}$meas and $\mathrm{I}_{\text {measoff, with ex- }}$ pectation as seen in Fig. 7 and in Table 4. In addition, a good agreement between the no-beam resonator response and the measurement offset from in-beam measurements confirms that cavity response and the measurement offset are uncorrelated.

For the resonator sensitivity, the higher expectation with respect to measurement, especially for energies $109 \mathrm{MeV}$ and $79 \mathrm{MeV}$ demands for an accurate correction for the beam losses. In other words, an absolute measure of the beam current and momentum spread is necessary to have good agreement of the sensitivity within one $\sigma$.

It is also important to remember that the measured sensitivity is valid only for the present location of the resonator i.e. $16 \mathrm{~m}$ from the degrader. Any relocation of the resonator requires for a new calibration for each individual energy. The need to calibrate individually at multiple energies can be replaced by single energy calibration with relocation of the resonator close to the degrader. This is because the bunch length elongation is affected to a lower extent by the energy spread from the degrader.

The calibration performed should be considered invariant of time since PROSCAN is a temperature controlled environment. Thus, reliable beam currents can be measured with the sensitivity derived from the measurements. It is important to remember, however, these measurements are relative since a calibration was performed due to the bunch length dependence.

Beam currents down to $0.15 \mathrm{nA}$ have been measured with $0.05 \mathrm{nA}$ resolution by the cavity resonator with the existing setup. Further performance improvements of this resonator are possible with design of the resonator as a single port or with improved electrical matching of the measurement port in the multiport resonator. Both methods are expected to improve the signal level by up to $50 \%$ for a given beam current which is of interest for the application in PROSCAN.

The cavity resonator can provide beam current information for the range $0.1-10 \mathrm{nA}$, it is delivered over an integration time of $1 \mathrm{~s}$. This limits the functionality of the resonator in patient treatments. Thus, the cavity resonator can be used only for triggering interlocks as a beam operation safety measure as well as controlling the operation parameters within the normal ranges.

However, in circumstances such as daily quality checks, high beam current irradiation as in FLASH etc. a cavity resonator is advantageous and could potentially replace ICs. Moreover, FLASH could pose a risk for ICs due to space charge effect that could result in a non-linear response from them. Thus, the use of the cavity resonator will relay all the necessary measures [9], required for a proper functioning of the ICs. Moreover, with the inclination towards proton FLASH therapy lately, a cavity resonator could be used during patient treatment since the beam currents are in the range of hundreds of $\mathrm{nA} \mathrm{[40].} \mathrm{This} \mathrm{would} \mathrm{lower} \mathrm{the}$ integration time to a few tens of microseconds enabling on-line beam monitoring.

\section{Conclusion}

In this work, we have demonstrated successful non-invasive beam current measurements at a proton therapy facility in the range $0.1-10 \mathrm{nA}$ for the energy range $238-70 \mathrm{MeV}$. This relative beam current measurement has been achieved with a resolution of $0.05 \mathrm{nA}$ with the help of a $\mathrm{TM}_{010}$ mode dielectric-filled reentrant cavity resonator. To our knowledge, this is the first time, non-invasive beam current measurement has been achieved at a proton therapy facility such as PROSCAN. These results indicate that cavity resonators have the potential to replace ICs for beam current measurements at proton therapy facilities.

\section{Funding}

This project received funding from the European Union's Horizon 2020 research and innovation programme under the Marie SklodowskaCurie grant agreement No. 675265.

\section{Declaration of Competing Interest}

The authors declare that they have no known competing financial interests or personal relationships that could have appeared to influence the work reported in this paper.

\section{Acknowledgments}

The authors would like to thank Kotrle Goran for construction of the prototype, the maintenance group and the vacuum group for installation of the prototype in the beamline.

\section{References}

[1] Schippers JM, Dölling R, Duppich J, Goitein G, Jermann M, Mezger A, et al. The SC cyclotron and beam lines of PSI's new protontherapy facility PROSCAN. Nucl Instruments Methods Phys Res Sect B Beam Interact Mater Atoms 2007;261:773-6. https://doi.org/10.1016/j.nimb.2007.04.052.

[2] Schippers JM, Duppich J, Goitein G, Jermann M, Lomax A, Pedroni E, et al. The use of protons in cancer therapy at PSI and related instrumentation. J Phys Conf Ser 2006;41:61-71. https://doi.org/10.1088/1742-6596/41/1/005.

[3] Kube G. Specific diagnostics needs for different machines. Cern Accel Sch Beam Diagnost 2008:1-64. https://doi.org/10.5170/CERN-2009-005. 
[4] Webber RC. Charged particle beam current monitoring tutorial. AIP Conf. Proc. 333, vol. 3, 2008, p. 3-23. https://doi.org/10.1063/1.48014.

[5] Dölling R. Profile, current, and halo monitors of the PROSCAN beam lines. AIP Conf Proc 2004;732:244-52. https://doi.org/10.1063/1.1831154.

[6] Duperrex PA. Latest diagnostic electronics development for the PROSCAN proton accelerator. AIP Conf Proc 2004;732:268-75. https://doi.org/10.1063/1.1831157.

[7] Strehl P. Beam Instrumentation and Diagnostics. 2006. https://doi.org/10.1007/3540-26404-3.

[8] Dölling R. Beam Diagnostics for Cyclotrons. Cyclotrons 2010 Proc, Vol. 19. Lanzhou, China: JACoW Publishing; 2010. p. 344-50.

[9] Dölling R. Ionisation chambers and secondary emission monitors at the PROSCAN beam lines. AIP Conf Proc 2006;868:271-80. https://doi.org/10.1063/1.2401414.

[10] Denard J-C. Beam Current Monitors. In: Brandt D, editor. CAS - Cern Accel. Sch. Course Beam Diagnostics, Dourdan, France: 2008, p. 141-55. https://doi.org/10. 5170/CERN-2009-005.

[11] Jing C, Shao J, Power J, Low YCA, SystemIPAC, CBPM. ninth Int. Part Accel Conf 2018;2018:7-9. https://doi.org/10.18429/JACoW-IPAC2018-WEPAF059.

[12] Forck P, Kowina P, Liakin D. CERN accelerator school beam diagnostics. Cern Eur Organ Nucl Res 2009. https://doi.org/10.5170/CERN-2009-005.

[13] Chao AW, Mess KH, Tigner M, Zimmermann F. Handbook of accelerator physics and engineering, second edition. Singapore: World Scientific; 2013. https://doi.org/10. 1142/8543.

[14] Odier P, Belohrad D, Gras J-J, Ludwig M. Operational experience and improvements of the LHC beam current transformers. In: Micahela Marx (DESY), Jan Chrin (PSI), Ian Martin (DIAMOND), Raphael Mueller (GSI) VRSc (GSI), editor. Proc. 10th Eur. Work. Beam diagnostics Instrum. Part. Accel., 2011.

[15] Stulle F, Bergoz J, Leemans WP, Nakamura K. Single pulse sub-picocoulomb charge measured by a turbo-ICT in a laser plasma accelerator. IBIC2016 Proc., JACoW Publishing; n.d., p. 119-22. https://doi.org/10.18429/JACoW-IBIC2016-MOPG35.

[16] Gasior M. An Inductive Pick-Up for Beam Position and Current Measurements. In: Peters A, Schaa VR, editors. DIPAC2003 Proc., Mainz, Germany: 2003, p. 53-5.

[17] Belohrad D. BEAM CHARGE MEASUREMENTS. In: Marx M, Chrin J, Martin I, Mueller R, SChaa VR, editors. Proc. DIPAC2011, Hamburg, Germany: 2011, p. 564-8.

[18] Gerigk F. Cavity types. CAS - Cern Accel. Sch. RF Accel., Ebeltoft: 2010, p. 277-98. https://doi.org/10.5170/CERN-2011-007.277.

[19] Keil B, Baldinger R, Ditter R, Engeler D, Koprek W, Kramert R, et al. Status of the SwissFEL BPM system. Proc 4th Int Beam Instrum Conf IBIC 2015 2015:497-501. https://doi.org/10.18429/JACoW-IBIC2015-TUPB065.

[20] Lorenz R. Cavity beam position monitors. AIP Conf Proc 1998;451:53-73. https:// doi.org/10.1063/1.57039.

[21] Simon C, Luong M, Chel S, Napoly O, Novo J, Roudier D, et al. Performance of a reentrant cavity beam position monitor. Phys Rev Spec Top - Accel Beams 2008;11:1-10. https://doi.org/10.1103/PhysRevSTAB.11.082802.

[22] Kim YI, Ainsworth R, Aryshev A, Boogert ST, Boorman G, Frisch J, et al. Cavity beam position monitor system for the accelerator test facility 2. Phys Rev Spec Top - Accel Beams 2012;15:1-16. https://doi.org/10.1103/PhysRevSTAB.15.042801.

[23] Srinivasan S, Duperrex P-A. Dielectric-filled reentrant cavity resonator as a low- intensity proton beam diagnostic. Instruments 2018;2:24. https://doi.org/10.3390/ instruments2040024.

[24] Srinivasan S, Duperrex PA. Reentrant Cavity Resonator for Low Intensities Proton Beam Measurements *. Proc IPAC2018, Vancouver, BC, Canada 2018:2341-4. https://doi.org/10.18429/JACoW-IPAC2018-WEPAL069.

[25] Macor Machinable Glass Ceramic Properties 2017. http://accuratus.com/ macormats.html.

[26] Alesini D. Power coupling. CAS - Cern Accel Sch RF Accel, Ebeltoft 2010:125-47. https://doi.org/10.5170/CERN-2011-007.125.

[27] Rizzoglio V, Adelmann A, Baumgarten C, Frey M, Gerbershagen A, Meer D, et al. Evolution of a beam dynamics model for the transport line in a proton therapy facility. Phys Rev Accel Beams 2017;20:1-12. https://doi.org/10.1103/ PhysRevAccelBeams.20.124702.

[28] Farley FJM. Optimum strategy for energy degraders and ionization cooling. Nucl Instrum Methods Phys Res Sect A Accel Spectromet Detect Assoc Equip 2005;540:235-44. https://doi.org/10.1016/j.nima.2004.11.034.

[29] Anferov V. Energy degrader optimization for medical beam lines. Nucl Instrum Methods Phys Res Sect A Accel Spectromet Detect Assoc Equip 2003;496:222-7. https://doi.org/10.1016/S0168-9002(02)01625-X.

[30] Petzoldt J, Roemer KE, Enghardt W, Fiedler F, Golnik C, Hueso-González F, et al. Characterization of the microbunch time structure of proton pencil beams at a clinical treatment facility. Phys Med Biol 2016;61:2432-56. https://doi.org/10. 1088/0031-9155/61/6/2432.

[31] Vavilov P. Ionization losses of high-energy heavy particles. Sov Phys JETP 1957;5:749-51.

[32] Van Goethem MJ, Van Der Meer R, Reist HW, Schippers JM. Geant4 simulations of proton beam transport through a carbon or beryllium degrader and following a beam line. Phys Med Biol 2009;54:5831-46. https://doi.org/10.1088/0031-9155/ 54/19/011.

[33] Shafer RE. Beam Position Monitoring. AIP Conf Proc 1990;212:26-58. https://doi. org/10.1063/1.39710.

[34] Johansen E. VME MESTRA Rev C Specification. 2020. https://doi.org/10.5281/ zenodo. 3887550

[35] R\&K-LA100-0S LOW NOISE AMPLIFIER n.d.:1000. http://www.rk-microwave. com/jp/products/pdf/LA101-0S_01.pdf (accessed January 16, 2020).

[36] FEMTO. Datasheet Ultra-Wideband Voltage Amplifier DUPVA-1-70 Variable-Gain n.d.:1-7. www.femto.de (accessed January 15, 2020).

[37] Mini-circuits. Low Noise Amplifier - S-Band n.d. https://www.minicircuits.com/ pdfs/ZX60-P103LN + .pdf (accessed January 16, 2020).

[38] ADC _ 3110 / 3111 - Eight Channel 16-bit ADC Data Sheet n.d. https://www.ioxos. ch/produit/adc-3110-3111/ (accessed January 15, 2020).

[39] Lipka D. Cavity Bpm Designs, Related Electronics and Measured Performances. Proc. DIPAC09, 2009, p. 280-4.

[40] Patriarca A, Fouillade C, Auger M, Martin F, Pouzoulet F, Nauraye C, et al. Experimental set-up for FLASH PROTON IRRADIATION OF SMALL ANIMALS USING A CLINICAL SYSTEm. Int J Radiat Oncol Biol Phys 2018. https://doi.org/10. 1016/j.ijrobp.2018.06.403. 